\title{
Increased plasma microfibrillar-associated protein 4 is associated with atrial fibrillation and more advanced left atrial remodelling
}

Xianlin Zhang ${ }^{1,2}$, Hailing $\mathrm{Li}^{1}$, Wenxin Kou${ }^{1}$, Kai Tang ${ }^{1}$, Dongdong Zhao', Jingying Zhang ${ }^{1}$, Jianhui Zhuang ${ }^{1}$, Yifan Zhao ${ }^{1}$, Shuya $\mathrm{Ji}^{1}$, Wenhui Peng ${ }^{1}$, Yawei Xu ${ }^{1}$

${ }^{1}$ Department of Cardiology, Shanghai Tenth People's Hospital of Nanjing Medical University; Shanghai Tenth People's Hospital, Tongji University School of Medicine, Shanghai, China

2Department of Cardiology, the People's Hospital of Maanshan, Maanshan City, Anhui Province, China

Submitted: 30 January 2018

Accepted: 14 March 2018

Arch Med Sci 2019; 15 (3): 632-640

DOI: https://doi.org/10.5114/aoms.2018.74953

Copyright (c) 2018 Termedia \& Banach

\section{Abstract}

Introduction: This study aimed to evaluate the relationship of plasma microfibrillar-associated protein 4 (MFAP4) to atrial fibrillation (AF) and atrial structural remodelling.

Material and methods: Plasma MFAP4 levels were measured in 92 patients with AF (61 paroxysmal AF (PAF) patients and 31 persistent AF (PersAF) patients) and 71 control subjects without AF. Linear and logistic multivariate regression analyses were performed to determine the potential value of MFAP4 for predicting the incidence of AF and left atrial size. Then, plasma and atrial protein levels of MFAP4 and its association with atrial fibrosis ratio were analysed in an atrial-specific fibrosis rat model.

Results: There were significant differences in MFAP4 levels based on clinical group, with a gradient from control $(1.71 \pm 0.53 \mathrm{ng} / \mathrm{ml})$ to PAF $(1.98 \pm 0.53 \mathrm{ng} /$ $\mathrm{ml})$ to PersAF $(2.09 \pm 0.76 \mathrm{ng} / \mathrm{ml})(p<0.01)$. With multivariate analyses, plasma MFAP4 was found to be an independent determinant of left atrial diameter in AF patients. In atrial fibrosis rats, both plasma MFAP4 and atrial MFAP4 protein levels increased in atrial fibrosis rats and positively correlated with atrial fibrosis severity.

Conclusions: Plasma MFAP4 was increased in patients with AF and was highest in those with PersAF; both plasma MFAP4 and atrial MFAP4 protein expression were directly associated with the extent of LA structural remodelling.

Key words: microfibrillar-associated protein 4, atrial fibrillation, atrial fibrosis, biomarker.

\section{Introduction}

Atrial fibrillation (AF) is the most common arrhythmia in the clinical setting and can induce several cardiovascular complications, including stroke, heart failure, and myocardial infarction [1-4], which results in increased mortality and public health costs [5]. Structural remodelling has been observed in both clinical and experimental AF paradigms and is an important feature of the AF substrate, producing fibrosis that alters atrial tissue composition and function. Atrial tissue fibrosis affect-

\author{
Corresponding authors: \\ Yawei Xu MD, PhD \\ Hailing Li MD, PhD \\ Department \\ of Cardiology \\ Shanghai Tenth \\ People's Hospital \\ Tongji University \\ School of Medicine \\ 301 Yanchang Road \\ Shanghai 200072, China \\ Phone: +862166307259 \\ Fax: +862166301051 \\ E-mail: xuyawei@tongji.edu.cn; \\ lihailin_2004@126.com
}


ing the left atrium is a major determinant of the progression of AF [6]; thus, a more extensively remodelled atrium represents the substrate needed for the arrhythmia to persist. A clear correlation has been shown between $A F$ and the degree of atrial fibrosis in post-mortem histological analysis [7]. Atrial fibrosis is also a major determinant for the success of rhythm control strategies in $A F$, including catheter ablation, one of the most efficacious methods of treating patients with AF [8]. Data on circulating fibrosis markers and AF are limited; however, markers of collagen turnover are associated with the type and duration of AF and as well as with postsurgical AF and AF recurrence after ablation [9].

Microfibrillar-associated protein 4 (MFAP4) is a matricellular protein belonging to the fibrinogen-related protein superfamily. This family also includes ficolins, fibroleukin, fibrinogen $\mathrm{C}$ domain containing 1 (FIBCD1), angiopoietins, and tenascins, which play multifaceted roles in innate immunity, the development of the cardiovascular system, and the normal functioning of the endothelium [10, 11]. MFAP4 has been reported to participate in elastic fibre formation [10, 12]. Although little is known about its molecular function, the relevance of MFAP4 is well-established in diseases associated with remodelling of the extracellular matrix (ECM), such as vascular stenosis [11], aortic aneurysms $[13,14]$, pulmonary hypertension [15], and hepatic fibrosis [16]. A previous study reported that MFAP4 mRNA was more highly expressed in the heart than in other elastic tissues [17], indicating that MFAP4 might play an important role in the process of cardiac fibrosis. It is also reported that serum MFAP4 varies in groups of patients with different coronary atherosclerotic disease [17]. However, the relationship between MFAP4 and the development of AF has not been studied. In this study we hypothesised that MFAP4 might reflect disease severity in patients with $A F$, with the plasma level reflecting the extent of left atrial structural remodelling. In a patient cohort with paroxysmal and persistent $A F$, and an atrial-specific fibrosis rat model, we aimed to evaluate the association of plasma MFAP4 with AF and atrial structural remodelling.

\section{Material and methods}

\section{Study population}

In this study, 92 symptomatic AF patients (61 paroxysmal AF (PAF) and 31 persistent AF (PerSAF)) and 71 control subjects hospitalised at the Department of Cardiology, Shanghai Tenth People's Hospital (China) from March to October 2016. Clinical classification of AF was based on expert consensus statements. All subjects with ischaemic heart disease, structural heart disease (including moderate-severe valvular disease, hypertrophic or dilated cardiomyopathy, and congenital heart disease), uncontrolled thyroid or parathyroid disease, chronic liver, kidney, or lung disease, systemic or local inflammatory or infectious disease, contraindication for anticoagulation, pregnancy, and malignancy were excluded from the study. The 71 control individuals had no history of AF or other significant supraventricular or ventricular arrhythmias, or of taking antiarrhythmic medication. Risk scores for stroke were evaluated by CHADS2 score (one point each for history of $\mathrm{HF}$, hypertension, age $\geq 75$ years, diabetes, two points for history of stroke, or transient ischaemic attack) [18] and CHA2DS2-VASc score (one point each for history of HF, hypertension, age 65 to 74 years, diabetes, vascular disease, female sex, and two points each for age $\geq 75$ years, stroke, or transient ischaemic attack) [19]. Written, informed consent was obtained from all subjects before their participation in the study. The study protocol was approved by the Shanghai Tenth Hospital Ethics Committee.

\section{Laboratory tests, electrocardiogram (ECG), and transthoracic echocardiogram (TTE)}

A comprehensive clinical assessment was performed, which included in-office blood pressure measurements, anthropometric measurements, and a TTE for assessment of left ventricular (LV) diastolic function and of myocardial deformation through two-dimensional speckle tracking echocardiography (2D-STE) (Vivid E9, GE Vingmed UItrasound AS, Norway). Vascular disease was identified through a previously established diagnosis of coronary artery disease, peripheral artery disease, carotid disease, renal artery disease, or aortic atheroma. Standard 12-lead ECG and echocardiography were performed in all subjects. In all groups, venous blood samples were drawn in tubes with ethylenediaminetetraacetic acid (EDTA) and centrifuged at $1000 \times g$ for $10 \mathrm{~min}$. Plasma was stored in $\mathrm{a}-80^{\circ} \mathrm{C}$ ultra-low-temperature refrigerator until biochemical analysis. As described previously [17], plasma MFAP4 levels were quantified using commercially available specific enzyme-linked immunosorbent assays (ELISAs) according to the manufacturer's protocol (Abbexa, abx252747, Cambridge, UK). The sensitivity of the MFAP4 ELISA was $0.188 \mathrm{ng} / \mathrm{ml}$, whereas the degree of precision of the ELISA system in terms of coefficient of variance (per cent) of intra-assay was between $1.38 \%$ and $1.84 \%$, and that of inter-assays was between $4.9 \%$ and $9.3 \%$. Spike recovery and linearity were in the range $97.7-100.8 \%$ and $100-$ $109 \%$, respectively. White blood cell count (WBC), C-reactive protein (CRP), N-terminal pro-brain natriuretic peptide (NT-proBNP), cardiac troponin 
(C-TNI), plasma levels of glucose, haemoglobin $\mathrm{A}_{1 \mathrm{c}}$ $\left(\mathrm{HbA}_{1 \mathrm{c}}\right)$ and lipid profiles, including total cholesterol (TC), triglycerides (TG), low-density lipoprotein cholesterol (LDL-C), and high-density lipoprotein cholesterol (HDL-C), were measured by colorimetric enzymatic assay systems (Roche MODULAR P-800, Switzerland).

\section{Atrial fibrosis rat animal model, western blots, and histological analysis}

Our group reported that the Myl4 E11K mutation had previously induced atrial-specific fibrosis in a rat model [20] (accepted and soon to be published). Rat left atrial samples for protein analysis were fresh frozen in liquid nitrogen, weighed on an electronic balance, and stored in liquid nitrogen until transfer into $\mathrm{a}-80^{\circ} \mathrm{C}$ freezer; all samples were stored at $-80^{\circ} \mathrm{C}$ before use. Rat samples for histological analysis were fixed in formaldehyde at room temperature and embedded in paraffin blocks. For western blots, samples of the atria were lysed in complete radioimmunoprecipitation assay (RIPA) buffer (10 mM Tris- $\mathrm{HCl}$ pH 7.4, $150 \mathrm{mM} \mathrm{NaCl}, 1 \% \mathrm{NP} 40,0.1 \%$ sodium dodecyl sulphate (SDS), $1 \mathrm{mM}$ phenylmethylsulfonyl fluoride (PMSF), 1× PhosStop and 1× protease inhibitor cocktail (Roche)) and homogenised by a Sonic Dismebrator 100 (Fisher Scientific). The protein concentration of tissue homogenates was measured using Bio-Rad Protein Assay, and equal amounts of soluble protein were separated on $12 \%$ polyacrylamide gels, transferred onto nitrocellulose membrane, and followed by routine western blot analysis. Primary antibodies were purchased from Santa Cruz (Vinculin sc-73614, MFAP4 sc-398438, Santa Cruz, CA, USA). Horseradish peroxidase (HRP)-conjugated secondary antibodies were purchased from BioRad Laboratories. For histological staining, sections of $5 \mu \mathrm{m}$ thickness were sliced. Left atrial fibrosis was obtained using a Modified Masson's Trichrome Stain Kit from Scy Tek Laboratories. For relative left atria fibrosis, 6 to 10 fields/ samples, and 4 to 5 samples/group were averaged.

\section{Statistical analysis}

Statistical analyses were performed using Statistical Package for Social Sciences (SPSS) for Windows 10. Numerical variables with a normal distribution were presented as the mean $\pm \mathrm{SE}$, numerical variables with a skewed distribution as the median, and categorical variables as percentages. For numerical variables, an independent sample $t$-test and the Mann-Whitney $U$ test were used for intergroup comparisons. The $\chi^{2}$ test and Fisher's exact tests were used for comparisons of categorical variables. The association between variables was evaluated with the Pearson rank correlation.
Subsequently, analysis was undertaken through multivariate logistic regression to assess a set of independent variable predictors of left atrial size. A two-tailed $p<0.05$ was considered statistically significant for all analyses.

\section{Results}

\section{Characteristics of subjects}

The mean age of the study population was $62.6 \pm 8.2$ years, and 78 (48\%) participants were male. Other demographic and clinical data are presented in Table I. Consistent with the atrial remodelling intrinsic to a history of $A F$, there were significant differences between the control and PAF groups in LA diameter (LAD). Typical of this group, the PersAF group had a significantly higher CHA2DS2-VASc score (older age, higher prevalence of hypertension, and worse liver and kidney function; $p<0.05$ for all comparisons), evidence of poorer LV systolic function, and more advanced LA structural remodelling, as well as more anti-arrhythmic drug usage (Table I).

\section{Plasma MFAP4 levels increased in AF patients and predicted AF occurrence}

The median plasma MFAP4 levels were 1.88 $\pm 0.65 \mathrm{ng} / \mathrm{ml}$, with an interquartile range of 1.44$2.24 \mathrm{ng} / \mathrm{ml}$. There were significant differences based on clinical group in MFAP4 levels, with a gradient from control $(1.71 \pm 0.53 \mathrm{ng} / \mathrm{ml})$ to PAF $(1.98$ $\pm 0.53 \mathrm{ng} / \mathrm{ml}$ ) to PersAF (2.09 $\pm 0.76 \mathrm{ng} / \mathrm{ml}$ ) (Figure 1). MFAP4 levels were highest in the PersAF group (PersAF vs. control $p=0.006$ and PersAF vs. PAF $p=0.032$ ) (Figure 1 ). To assess the potential ability of MFAP4 in the prediction for AF occurrence, we performed multivariate analyses, and the results showed that a high plasma MFAP4 concentration was an independent determinant of $\mathrm{AF}$ (odds ratio $(\mathrm{OR})=4.259,95 \%$ confidence interval (CI): 1.092, 16.608) in the total population (Table II). The results suggest that plasma MFAP4 has potential value in the prediction of AF occurrence.

\section{Relationship of plasma MFAP4 to the left atrial size}

Using Pearson correlation analysis (Table III), plasma MFAP4 levels were correlated with increasing age, CRP, NT-proBNP and LAD, a history of hypertension or $A F$, and a higher CHA2DS2VASc score. By univariate regression (Table IV), increased plasma MFAP4 was also associated with increasing age, TG, CRP, and LAD.

Of note, plasma MFAP4 levels were positively associated with LA structural remodelling manifested by elevated LA size. Moreover, multiple 
Table I. Baseline characteristics of the study population

\begin{tabular}{|c|c|c|c|c|c|}
\hline $\begin{array}{l}\text { Clinical characteristics } \\
\text { (Total } n=163 \text { ) }\end{array}$ & $\begin{array}{l}\text { Control } \\
(n=71)\end{array}$ & $\begin{array}{l}\text { Paroxysmal AF } \\
\quad(n=61)\end{array}$ & $P$-value & $\begin{array}{l}\text { Persistent AF } \\
\quad(n=31)\end{array}$ & $P$-value \\
\hline Male, $n(\%)$ & $28(39.4)$ & $35(57.4)$ & 0.040 & $15(48.4)$ & 0.120 \\
\hline Age [years] & $62.24 \pm 0.88$ & $62.41 \pm 1.34$ & 0.909 & $64.00 \pm 1.21$ & 0.614 \\
\hline $\mathrm{SBP}[\mathrm{mm} \mathrm{Hg}]$ & $128 \pm 2$ & $128 \pm 2$ & 0.835 & $126 \pm 3$ & 0.882 \\
\hline $\mathrm{DBP}[\mathrm{mm} \mathrm{Hg}]$ & $75 \pm 1$ & $77 \pm 1$ & 0.229 & $73 \pm 2$ & 0.202 \\
\hline $\mathrm{HR}$ [bpm] & $79 \pm 1$ & $79 \pm 2$ & 0.958 & $86 \pm 2$ & 0.023 \\
\hline BMI $\left[\mathrm{kg} / \mathrm{m}^{2}\right]$ & $23.43 \pm 0.34$ & $24.95 \pm 0.49$ & 0.023 & $23.98 \pm 1.16$ & 0.074 \\
\hline Smoker, $n(\%)$ & $17(23.9)$ & $25(40.9)$ & 0.036 & $8(25.8)$ & 0.086 \\
\hline Hypertension, $n$ (\%) & $1(1.4)$ & $9(14.8)$ & 0.004 & $9(29.0)$ & $<0.001$ \\
\hline Diabetes mellitus, $n(\%)$ & $1(1.4)$ & $2(3.3)$ & 0.472 & $0(0)$ & 0.508 \\
\hline Hyperlipidaemia, $n(\%)$ & $2(2.8)$ & $3(4.9)$ & 0.528 & $0(0)$ & 0.428 \\
\hline Statin therapy, $n(\%)$ & $2(2.8)$ & $10(16.4)$ & 0.007 & $8(25.8)$ & 0.081 \\
\hline Amiodarone therapy, $n(\%)$ & $0(0)$ & $11(18.0)$ & $<0.001$ & $0(0)$ & $<0.001$ \\
\hline Propafenone therapy, $n(\%)$ & $0(0)$ & $11(18.0)$ & $<0.001$ & $0(0)$ & $<0.001$ \\
\hline$\beta$-Blocker therapy, $n(\%)$ & $0(0)$ & $21(34.4)$ & $<0.001$ & $13(41.9)$ & $<0.001$ \\
\hline $\mathrm{PR}[\mathrm{ms}]$ & $160.92 \pm 2.60$ & $164.70 \pm 4.98$ & 0.461 & - & - \\
\hline $\mathrm{QT}[\mathrm{ms}]$ & $399.08 \pm 3.80$ & $399.08 \pm 6.75$ & 1.000 & $371.16 \pm 5.91$ & 0.004 \\
\hline QT-C [ms] & $420.10 \pm 6.73$ & $432.00 \pm 3.96$ & 0.118 & $436.10 \pm 4.57$ & 0.143 \\
\hline CRP $[\mathrm{mg} / \mathrm{l}]$ & $3.3(3.3,3.3)$ & $3.3(3.3,3.3)$ & 0.160 & $3.3(3.3,3.3)$ & 0.306 \\
\hline WBC $\left[\times 10^{9} / 1\right]$ & $5.62 \pm 0.18$ & $6.14 \pm 0.22$ & 0.055 & $5.60 \pm 0.19$ & 0.111 \\
\hline $\mathrm{PLT}\left[\times 10^{9} / \mathrm{I}\right]$ & $218.13 \pm 7.17$ & $201.30 \pm 8.02$ & 0.116 & $187.50 \pm 10.73$ & 0.055 \\
\hline Glucose [mmol/l] & $4.99 \pm 0.07$ & $5.00 \pm 0.11$ & 0.966 & $5.40 \pm 0.23$ & 0.071 \\
\hline $\mathrm{TC}[\mathrm{mmol} / \mathrm{l}]$ & $4.82 \pm 0.19$ & $4.39 \pm 0.11$ & 0.051 & $4.19 \pm 0.15$ & 0.038 \\
\hline TG [mmol/l] & $1.41 \pm 0.09$ & $1.56 \pm 0.16$ & 0.374 & $1.31 \pm 0.09$ & 0.480 \\
\hline LDL-C [mmol/l] & $2.86 \pm 0.09$ & $2.65 \pm 0.08$ & 0.108 & $2.58 \pm 0.13$ & 0.132 \\
\hline ALT [U/I] & $14.98 \pm 0.69$ & $20.17 \pm 1.91$ & 0.021 & $23.14 \pm 3.35$ & 0.007 \\
\hline AST [U/I] & $18.94 \pm 0.63$ & $22.02 \pm 1.97$ & 0.148 & $26.12 \pm 2.79$ & 0.024 \\
\hline BUN [mmol/l] & $5.38 \pm 0.15$ & $5.87 \pm 0.18$ & 0.039 & $5.99 \pm 0.25$ & 0.045 \\
\hline $\mathrm{Cr}[\mu \mathrm{mol} / \mathrm{l}]$ & $65.97 \pm 1.52$ & $73.49 \pm 2.35$ & 0.005 & $68.29 \pm 2.41$ & 0.019 \\
\hline $\mathrm{UA}[\mu \mathrm{mol} / \mathrm{l}]$ & $352.60 \pm 58.73$ & $334.56 \pm 12.49$ & 0.756 & $373.59 \pm 17.33$ & 0.144 \\
\hline NT-proBNP [pg/ml] & $54.1(32.5,89.9)$ & $205.4(66.9,582.5)$ & $<0.001$ & $800.2(464.5,1212.0)$ & $<0.001$ \\
\hline C-TNI [ng/ml] & $0.005(0.004,0.007)$ & $0.007(0.004,0.011)$ & 0.038 & $0.008(0.006,0.013)$ & 0.003 \\
\hline FT3 [pmol/l] & $4.48(4.22,4.78)$ & $4.73(4.07,5.03)$ & 0.186 & $4.81(4.49,5.16)$ & 0.098 \\
\hline FT4 [pmol/l] & $13.76(12.54,15.11)$ & $15.29(14.05,17.06)$ & 0.444 & $14.93(13.39,16.43)$ & 0.336 \\
\hline TSH [mIU/l] & $1.91(1.44,3.44)$ & $2.11(1.41,3.02)$ & 0.721 & $2.11(1.40,4.50)$ & 0.650 \\
\hline CHADS2 & $0.00(0.00,0.00)$ & $0.00(0.00,0.00)$ & $<0.001$ & $1.00(0.00,2.00)$ & $<0.001$ \\
\hline CHA2DS2-VASC & $1.00(1.00,1.00)$ & $1.10(0.00,1.00)$ & 0.406 & $2.00(1.00,3.00)$ & 0.003 \\
\hline MFAP4 [ng/ml] & $1.71 \pm 0.06$ & $1.97 \pm 0.09$ & 0.021 & $2.09 \pm 0.14$ & 0.009 \\
\hline LAD [mm] & $35.74 \pm 0.57$ & $40.46 \pm 0.77$ & $<0.001$ & $47.20 \pm 1.11$ & $<0.001$ \\
\hline LVDd [mm] & $43.79 \pm 0.53$ & $45.07 \pm 0.75$ & 0.155 & $47.03 \pm 0.91$ & 0.014 \\
\hline LVEF (\%) & $66.00 \pm 0.59$ & $63.63 \pm 0.83$ & 0.022 & $60.59 \pm 1.20$ & $<0.001$ \\
\hline
\end{tabular}

$S B P$ - systolic blood pressure, DBP - diastolic blood pressure, HR - heart rate, BMI - body mass index, TC - total cholesterol, TG - triglyceride, $L D L-C$-low-density lipoprotein cholesterol, CRP-C-reactive protein, WBC-white blood cell count, PLT-platelet, ALT-alanine aminotransferase, $A S T$ - aspartate aminotransferase, BUN - blood urea nitrogen, $\mathrm{Cr}$ - serum creatinine concentrations, UA - uric acid, NT-proBNP - N-terminal pro-brain natriuretic peptide, C-TNI - cardiac troponin, FT3 - free triiodothyronine, FT4 - free thyroxine, TSH - thyroid stimulating hormone, MFAP4 - microfibrillar-associated protein 4, LAD - left atrial diameter, LVDd - left ventricular end-diastolic dimension, LVEF - left ventricular ejection fraction. Data are presented as median (interquartile range) or mean value $\pm S E$. The $p$-value in column 4 refers to the comparison between the control and paroxysmal AF groups. The p-value in column 6 refers to the overall comparison between the control, paroxysmal, and persistent AF groups. 
A

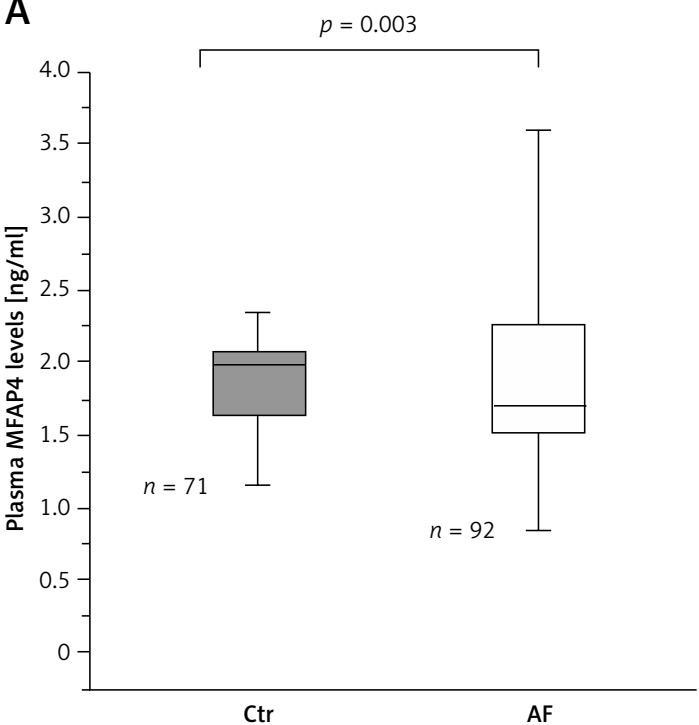

B

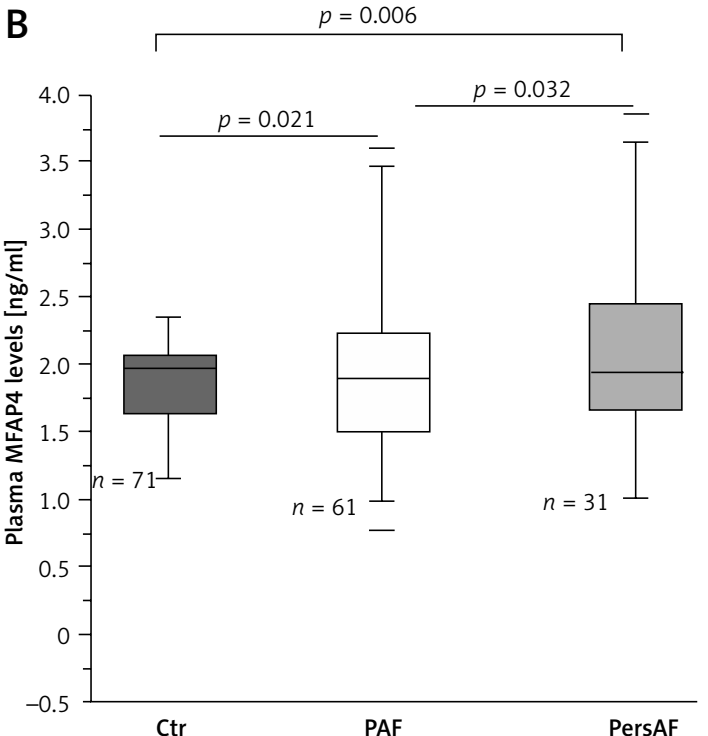

Figure 1. Plasma MFAP4 levels in different groups. A - Plasma MFAP4 levels were higher in AF patients compared with control (Ctr) individuals. B - There were significant differences based on clinical group in MFAP4 levels, with a gradient from control to PAF and thence PersAF. MFAP4 levels were highest in the PersAF group

Table II. Logistic regression analysis with the presence of $A F$

\begin{tabular}{|lccc|}
\hline Variable & OR & $P$-value & $95 \% \mathrm{Cl}$ \\
\hline Age & 1.135 & 0.006 & $(1.038,1.241)$ \\
\hline MFAP4 & 4.259 & 0.037 & $(1.092,16.608)$ \\
\hline PLT & 1.014 & 0.016 & $(1.003,1.026)$ \\
\hline LDL-C & 1.226 & 0.629 & $(0.536,2.803)$ \\
\hline FT3 & 1.735 & 0.386 & $(0.499,6.032)$ \\
\hline FT4 & 1.928 & $<0.001$ & $(1.362,2.728)$ \\
\hline LAD & 1.391 & $<0.001$ & $(1.201,1.610)$ \\
\hline
\end{tabular}

The same as in Table I; only numeric variables were listed in the table. Adjusted confounders: gender, hypertension, diabetes mellitus, hyperlipidaemia, statin therapy; $\mathrm{Cl}$ - confidence interval. regression analyses revealed that for $\mathrm{AF}$ patients, MFAP4, along with body mass index (BMI) and NT-proBNP, effectively predicted LAD after adjustment for age and hypertension $\left(R^{2}=0.543\right.$, $p<0.001$ ) (Table V).

MFAP4 was increased in fibrosis rat atrium and associated with the fibrosis severity: data from atrial fibrosis rat model

Clinical data indicate that plasma MFAP4 is positively associated with LA structural remodelling. We confirmed this in a previously constructed atrial-specific fibrosis rat model [20]. Data showed that plasma MFAP4 levels also increased in atrial fibrosis rats compared with wild-type rats, and

Table III. Pearson correlation analysis of MFAP4 and clinical parameters

\begin{tabular}{|c|c|c|c|c|c|c|}
\hline \multirow[t]{2}{*}{ Parameter } & \multicolumn{2}{|c|}{ MFAP4 in total $(n=163)$} & \multicolumn{2}{|c|}{ MFAP4 in AF $(n=92)$} & \multicolumn{2}{|c|}{ MFAP4 in control $(n=71)$} \\
\hline & $r$ & $P$-value & $r$ & $P$-value & $r$ & $P$-value \\
\hline AF & 0.229 & 0.003 & - & - & - & - \\
\hline CHADS2 & 0.151 & 0.055 & 0.097 & 0.358 & -0.095 & 0.430 \\
\hline CHA2DS2-VASC & 0.265 & 0.001 & 0.276 & 0.008 & 0.102 & 0.396 \\
\hline NT-proBNP & 0.294 & $<0.001$ & 0.256 & 0.017 & 0.208 & 0.091 \\
\hline LAD & 0.253 & 0.001 & 0.243 & 0.021 & 0.002 & 0.985 \\
\hline Age & 0.273 & $<0.001$ & 0.336 & 0.001 & 0.174 & 0.146 \\
\hline HBP & 0.174 & 0.026 & 0.163 & 0.120 & -0.105 & 0.382 \\
\hline CRP & 0.162 & 0.052 & 0.225 & 0.036 & -0.143 & 0.292 \\
\hline AST & 0.138 & 0.081 & 0.073 & 0.489 & 0.280 & 0.020 \\
\hline TG & 0.027 & 0.741 & -0.079 & 0.464 & 0.246 & 0.042 \\
\hline
\end{tabular}

Abbreviations: The same as in Table I. 
positively correlated with the atrial fibrosis severity (Figure 2). We also detected the MFAP4 protein levels in those fibrotic atria. Furthermore, data showed that MFAP4 protein was also increased in atrial fibrosis rats compared with wild-type rats (Figure 2).

\section{Discussion}

The key finding of this study was that plasma MFAP4 levels increased in patients with AF and were highest in those with PersAF. Pearson correlation analysis indicated that plasma MFAP4 levels were correlated with factors associated with PersAF, including older age, hypertension, heart failure (measured by NT-proBNP), higher CHA2DS2-VASc score, and left atrial size. Furthermore, multiple regression analyses revealed that increased plasma MFAP4 levels were directly associated with more advanced atrial structural remodelling, demonstrated through echocardiography LA size. Moreover, using an atrial-specific fibrosis rat model, we confirmed the correlation of MFAP4 with atrial remodelling.

The pathophysiology of AF is complex, involving the role of inflammation and structural remodelling with apoptosis and fibrosis [21, 22]. Biomarkers in human AF have been extensively studied in order to identify biomarkers that are useful for predicting the extent of atrial structural remodelling and the response to therapeutic interventions. Markers of a pro-thrombotic state, including platelet activation, enhanced thrombin generation, and endothelial dysfunction, have been associated with the duration and burden of $\operatorname{AF}[23,24]$. In addition to plasma biomarkers, imaging-based markers, such as cardiac magnetic resonance imaging, have been used to identify atrial myocardial fibrosis. Such techniques have demonstrated that the extent of structural remodelling at the time of AF ablation strongly predicts the likelihood of both maintaining sinus rhythm and significant reverse remodelling post-ablation [25]. These data highlight the importance of identifying a circulating biomarker with which to accurately identify the extent of atrial structural remodelling. To our knowledge, there are no previous reports relating plasma MFAP4 levels either to the extent of structural remodelling or to clinical outcomes in patients with AF.

The production of atrial fibrosis disrupts muscle-bundle continuity and causes AF-promoting conduction abnormalities [26]. Rapidly firing atrial cardiomyocytes produce substances that activate fibroblasts, providing a link between PAF episodes and fibrosis development [27]. MFAP4, produced by activated myofibroblasts, is a ubiquitous protein playing a potential role in ECM turnover. The MFAP4 protein is a disulphide-linked dimer that
Table IV. Univariate linear regression analysis of plasma MFAP4 levels with clinical variables

\begin{tabular}{|c|c|c|c|}
\hline Variable & $\beta$ Coefficient & $t$ & $P$-value \\
\hline $\begin{array}{l}\text { Clinical group } \\
\text { (control, PAF, } \\
\text { PersAF) }\end{array}$ & -0.055 & -0.479 & 0.633 \\
\hline Age & 0.348 & 3.153 & 0.002 \\
\hline Female gender & 0.025 & 0.161 & 0.873 \\
\hline Hypertension & -0.122 & -1.154 & 0.252 \\
\hline Diabetes & -0.211 & -1.749 & 0.085 \\
\hline Smoking & -0.139 & -0.919 & 0.361 \\
\hline BMI & -0.110 & -0.901 & 0.371 \\
\hline TC & -0.822 & -1.157 & 0.251 \\
\hline TG & 0.475 & 2.110 & 0.038 \\
\hline HDL & 0.447 & 1.790 & 0.078 \\
\hline LDL & 0.688 & 1.115 & 0.269 \\
\hline ALT & 0.067 & 0.586 & 0.560 \\
\hline Glucose & 0.060 & 0.506 & 0.614 \\
\hline CRP & 0.295 & 2.696 & 0.009 \\
\hline NT-proBNP & -0.099 & -0.645 & 0.521 \\
\hline LAD & 0.293 & 1.806 & 0.045 \\
\hline
\end{tabular}

Abbreviations: The same as in Table l; Cl - confidence interval.

Table V. Standard multiple regression analysis of $L A D$ in AF patients

\begin{tabular}{|lccc|}
\hline $\begin{array}{l}\text { Independent } \\
\text { variables }\end{array}$ & $\beta$ Coefficient & 95\% Cl & $P$-value \\
\hline \begin{tabular}{l} 
LAD $\left(R^{2}=0.543, F=9.758, p<0.001\right):$ \\
\hline MFAP4
\end{tabular} 0.266 & $(0.235,5.034)$ & 0.032 \\
\hline BMI & 0.303 & $(0.167,0.975)$ & 0.007 \\
\hline NT-proBNP & 0.563 & $(0.004,0.010)$ & $<0.001$ \\
\hline
\end{tabular}

Abbreviations: The same as in Table l; Cl - confidence interval.

forms higher oligomeric structures and its involvement has been suggested in elastic fibre formation [28]. A previous study reported that MFAP4 mRNA was more highly expressed in the heart than in other elastic tissues [17]. Our data also showed that MFAP4 protein highly expressed in the atrium and increased in fibrotic left atria, which indicated that MFAP4 might play an important role in the process of atrial fibrosis. In this study, with the use of a patient cohort with PAF and PersAF, we found that plasma MFAP4 levels were increased in AF, especially in PersAF patients. Multiple regression analyses also revealed that plasma MFAP4 levels were directly associated with the extent of LA size. This means that plasma MFAP4 may be a good biomarker to identify atrial myocardial remodelling. To confirm the link of MFAP4 to atrial struc- 
A

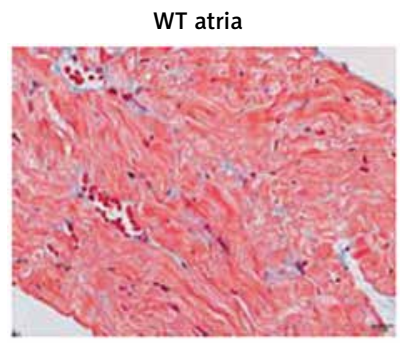

B

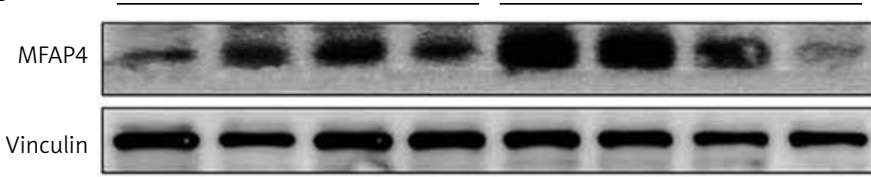

D

C

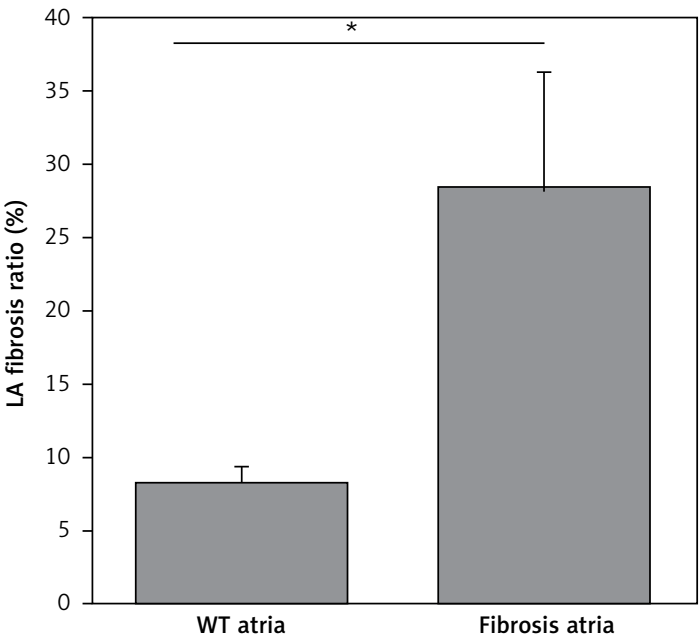

E

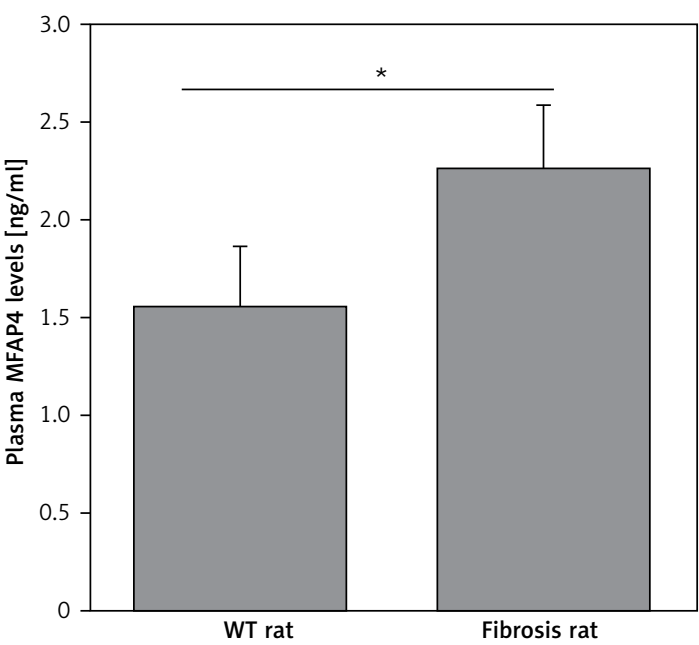

$\mathrm{F}$
Fibrosis atria

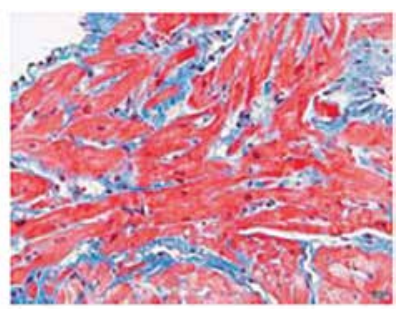

$\sim 29 \mathrm{kDa}$

$\sim 117 \mathrm{kDa}$
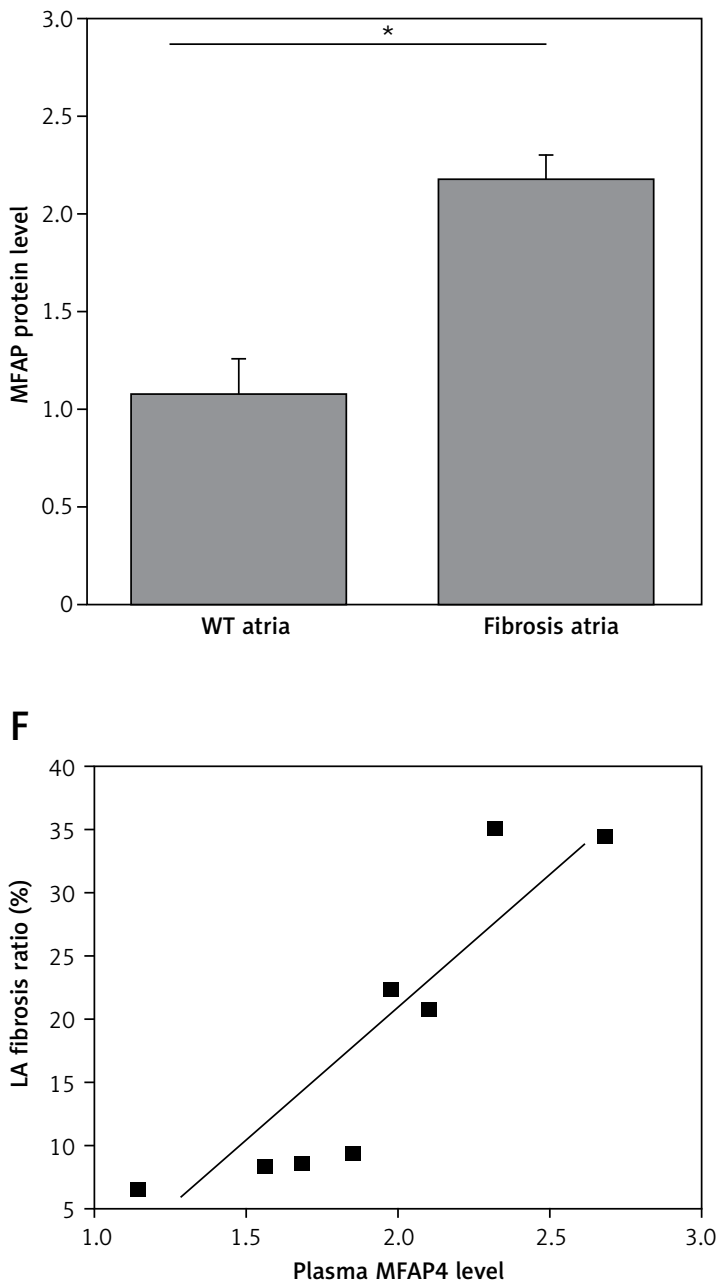

Figure 2. MFAP4 is increased in fibrosis rat atrium and associated with the fibrosis severity. A, C - Left atrial fibrosis was obtained using Modified Masson's Trichrome Stain. For relative left atria fibrosis, 6-10 fields/samples, and 4-5 samples/group were averaged. B, D - Western blots and mean data for MFAP4 protein expression in atria from WT and atrial fibrosis rats. E, F - Plasma MFAP4 levels also increased in atrial fibrosis rats and positively correlated with the atrial fibrosis severity. Data are mean \pm SEM. Protein from 4 independent rat atrial samples was subjected to immunoblot for each groups. ${ }^{*} p<0.05$ 
tural remodelling, we further used an atrial-specific rat model. It also showed that plasma MFAP4 was increased in atrial fibrosis rats and was positively correlated with the atrial fibrosis severity. The atrial remodelling level was associated with successful radiofrequency catheter ablation in patients with PersAF. With plasma MFAP4 levels increasing with more advanced remodelling and a PersAF phenotype, it might be expected that patients with AF but low plasma MFAP4 levels may benefit mostly from catheter ablation.

The MFAP4 gene consists of a signal peptide, a short N-terminal region comprising an Arg-GlyAsp sequence followed by the C-terminus. The Arg-Gly-Asp sequence is the ligand motif for cell surface integrins and is associated with cell adhesive activity [29]. On the other hand, MFAP4 has considerable sequence homology with the 36-kDa bovine microfibril-associated glycoprotein (MAGP-36), which was first discovered in porcine aorta and has since been detected in a wide variety of elastic tissues [30]. Both MFAP4 and MAGP-36 bind to elastin and collagen fibres [28]. Elastic fibres and collagen fibres are components of the ECM that ensure its structural integrity by maintaining the elasticity in the arterial wall, lungs, skin, and other dynamic connective tissues [31]. However, the mechanism by which MFAP4 participates in ECM turnover is still not fully understood. Although we found the MFAP4 protein was highly expressed in the atrium, a study has reported that in the myocardium, MFAP4 was intensely detected in elastic fibres located within blood vessels and the surrounding connective tissue, and no staining was seen in cardiomyocytes [17]. This suggested that MFAP4 exerted its function through the cardiac interstitial cells rather than cardiomyocytes. Further studies should be directed at investigating the mechanism of MFAP4 in atrial fibrosis and establishing a potential therapeutic role for MFAP4 in ameliorating atrial remodelling.

This study has several limitations that could not be eliminated. First of all, the sample size was small, and larger cohorts of patients with AF and control individuals are needed to extensively evaluate MFAP4 as a potential biomarker. Secondly, this is a cohort but not cross-sectional study, which would introduce biases to data analysis and prevent us from establishing a cause-effect relationship of MFAP4 with AF. Nevertheless, using an atrial-specific fibrosis rat model, we confirmed the correlation of MFAP4 with atrial remodelling.

In conclusion, using a patient cohort with PAF and PersAF, and an atrial-specific fibrosis rat model, we have shown that plasma MFAP4 was increased in patients with AF and highest in those with PersAF. Moreover, both plasma MFAP4 and atrial MFAP4 protein expression were directly as- sociated with the extent of LA structural remodelling.

\section{Acknowledgments}

This work was supported by the Chinese National Natural Science Foundation (Nos. 81470394, 81370391), Shanghai Sailing Program (No. 15YF1409400) and Fundamental Research Funds for Central Universities (No. 1501219105).

\section{Conflict of interest}

The authors declare no conflict of interest.

\section{References}

1. Begg GA, Holden AV, Lip GY, Plein S, Tayebjee MH. Assessment of atrial fibrosis for the rhythm control of atrial fibrillation. Int J Cardiol 2016; 220: 155-61.

2. Vlachos K, Letsas KP, Korantzopoulos P, et al. Prediction of atrial fibrillation development and progression: current perspectives. World J Cardiol 2016; 26: 267-76.

3. O'Neal WT, Qureshi W, Zhang ZM, Soliman EZ. Bidirectional association between atrial fibrillation and congestive heart failure in the elderly. J Cardiovasc Med (Hagerstown) 2016; 17: 181-6.

4. Lasek-Bal A, Gasior Z. Cardiovascular diseases in patients 65 years and younger with non-cardiogenic stroke. Arch Med Sci 2016; 12: 556-62.

5. Kim MH, Johnston SS, Chu BC, Dalal MR, Schulman KL. Estimation of total incremental health care costs in patients with atrial fibrillation in the United States. Circ Cardiovasc Qual Outcomes 2011; 4: 313-20.

6. Allessie M, Ausma J, Schotten U. Electrical, contractile and structural remodeling during atrial fibrillation. Cardiovasc Res 2002; 54: 230-46.

7. Smith JG, Newton-Cheh C, Almgren P, et al. Assessment of conventional cardiovascular risk factors and multiple biomarkers for the prediction of incident heart failure and atrial fibrillation. J Am Coll Cardiol 2010; 56: 1712-9.

8. Wazni OM, Marrouche NF, Martin DO, et al. Radiofrequency ablation vs antiarrhythmic drugs as first-line treatment of symptomatic atrial fibrillation: a randomized trial. JAMA 2005; 293: 2634-40.

9. Oakes RS, Badger TJ, Kholmovski EG, et al. Detection and quantification of left atrial structural remodeling with delayed-enhancement magnetic resonance imaging in patients with atrial fibrillation. Circulation 2009; 119: 1758-67.

10. Kasamatsu S, Hachiya A, Fujimura T, et al. Essential role of microfibrillar-associated protein 4 in human cutaneous homeostasis and in its photoprotection. Sci Rep 2011; 1: 164.

11. Schlosser A, Pilecki B, Hemstra LE, et al. MFAP4 promotes vascular smooth muscle migration, proliferation and accelerates neointima formation. Arterioscler Thromb Vasc Biol 2016; 36: 122-33.

12. Pilecki B, Holm AT, Schlosser A, et al. Characterization of microfibrillar-associated protein 4 (MFAP4) as a tropoelastin- and fibrillin-binding protein involved in elastic fiber formation. J Biol Chem 2016; 291: 1103-14.

13. Pilop C, Aregger F, Gorman RC, et al. Proteomic analysis in aortic media of patients with Marfan syndrome reveals increased activity of calpain 2 in aortic aneurysms. Circulation 2009; 120: 983-91. 
14. Modrego J, Lopez-Farre AJ, Martinez-Lopez I, et al. Expression of cytoskeleton and energetic metabolism-related proteins at human abdominal aortic aneurysm sites. J Vasc Surg 2012; 55: 1124-33.

15. Abdul-Salam VB, Wharton J, Cupitt J, et al. Proteomic analysis of lung tissues from patients with pulmonary arterial hypertension. Circulation 2010; 122: 2058-67.

16. Bracht T, Molleken C, Ahrens M, et al. Evaluation of the biomarker candidate MFAP4 for non-invasive assessment of hepatic fibrosis in hepatitis C patients. J Transl Med 2016; 14: 201.

17. Wulf-Johansson H, Lock Johansson S, Schlosser A, et al. Localization of microfibrillar-associated protein 4 (MFAP4) in human tissues: clinical evaluation of serum MFAP4 and its association with various cardiovascular conditions. PLoS One 2013; 8: e82243.

18. Gage BF, Waterman AD, Shannon W, et al. Validation of clinical classification schemes for predicting stroke: results from the National Registry of Atrial Fibrillation. JAMA 2001; 285: 2864-70.

19. Lip GY, Nieuwlaat R, Pisters R, Lane DA, Crijns HJ. Refining clinical risk stratification for predicting stroke and thromboembolism in atrial fibrillation using a novel risk factor-based approach: the euro heart survey on atrial fibrillation. Chest 2010; 137: 263-72.

20. Peng W, Li M, Li H, et al. Dysfunction of myosin lightchain 4 (MYL4) leads to heritable atrial cardiomyopathy with electrical, contractile, and structural components: evidence from genetically-engineered rats. J Am Heart Assoc 2017; 6: pii: e007030.

21. Vilchez JA, Roldan V, Hernandez-Romero D, et al. Biomarkers in atrial fibrillation: an overview. Int J Clin Pract 2014; 68: 434-43.

22. Yang H, Wu C, Xiao Y, Zhou S. Connexin and fibrosis related microRNAs in complex fractionated atrial electrograms. Arch Med Sci 2015; 11: 679-82.

23. Sohara H, Amitani S, Kurose M, Miyahara K. Atrial fibrillation activates platelets and coagulation in a time-dependent manner: a study in patients with paroxysmal atrial fibrillation. J Am Coll Cardiol 1997; 29: 106-12.

24. Li-Saw-Hee FL, Blann AD, Gurney D, Lip GY. Plasma von Willebrand factor, fibrinogen and soluble P-selectin levels in paroxysmal, persistent and permanent atrial fibrillation. Effects of cardioversion and return of left atrial function. Eur Heart J 2001; 22: 1741-7.

25. Tops LF, Delgado V, Bertini $M$, et al. Left atrial strain predicts reverse remodeling after catheter ablation for atrial fibrillation. J Am Coll Cardiol 2011; 57: 324-31.

26. Nattel S, Dobrev D. Electrophysiological and molecular mechanisms of paroxysmal atrial fibrillation. Nat Rev Cardiol 2016; 13: 575-90.

27. Burstein B, Qi XY, Yeh YH, Calderone A, Nattel S. Atrial cardiomyocyte tachycardia alters cardiac fibroblast function: a novel consideration in atrial remodeling. Cardiovasc Res 2007; 76: 442-52.

28. Schlosser A, Thomsen T, Shipley JM, et al. Microfibril-associated protein 4 binds to surfactant protein A (SP-A) and colocalizes with SP-A in the extracellular matrix of the lung. Scand J Immunol 2006; 64: 104-16.

29. Molleken C, Poschmann G, Bonella F, et al. MFAP4: a candidate biomarker for hepatic and pulmonary fibrosis? Sarcoidosis Vasc Diffuse Lung Dis 2016; 33: 41-50.

30. Toyoshima T, Ishida T, Nishi N, et al. Differential gene expression of $36-\mathrm{kDa}$ microfibril-associated glycoprotein (MAGP-36/MFAP4) in rat organs. Cell Tissue Res 2008; 332: $271-8$.

31. Kielty CM, Sherratt MJ, Shuttleworth CA. Elastic fibres. J Cell Sci 2002; 115: 2817-28. 